\title{
Predicting Perceptual Events Activates Corresponding Motor Schemes in Lateral Premotor Cortex: An fMRI Study
}

\author{
Ricarda I. Schubotz and D. Yves von Cramon \\ Max Planck Institute of Cognitive Neuroscience, D-04303 Leipzig, Germany
}

Received May 15, 2001

\begin{abstract}
The ability to recognize sequential patterns of external events enables us to predict their future course and thus to plan and execute actions based on current perceptions and previous experiences. Here we show with functional magnetic resonance imaging that even in the absence of movement the prediction of sequential patterns activates brain areas involved in the representation of specific motor schemas. Particularly, the prediction of size engages premotor areas involved in hand movements (superior part of the ventrolateral premotor cortex), whereas the prediction of pitch engages premotor areas involved in articulation (inferiormost ventrolateral premotor cortex). The findings indicate that events are mapped onto somatotopically corresponding motor schemes whenever we predict sequential perceptions. 2002 Elsevier Science (USA)
\end{abstract}

\section{INTRODUCTION}

In order to adapt our behavior to a continuously changing environment, we often have to set up perceptual expectations about ongoing sequential events. This anticipation optimizes the accuracy and speed of sensory processing and facilitates the preparation of appropriate motor responses. Sequential anticipation and prediction allow one, for instance, to track regular target motion with minimal phase lag in smooth pursuit eye movement (Lekwuwa and Barnes, 1996; Kawashima et al., 1998) or sensory-guided manual responses (Zhuang et al., 1998; Patel and Balaban, 2000).

A strong coupling between sequential perceptual prediction and sequential motor planning is also postulated in functional models of the brain, particularly of the frontal lobes. These are suggested to be involved in the sequential organization of behavior, housing "serial action programs" or "memories of the future" (Ingvar, 1985). Used as templates for extracting meaningful sequential information from sensory inputs, sequential programs are proposed to form the basis both for perceptual prediction and for goal-directed motor planning as a common substrate (Hommel et al., 2002). This view now gains new evidence from functional magnetic resonance imaging (fMRI) (Schubotz et al., 2000; Schubotz and von Cramon, 2001a,b). Particularly, it has been proposed that sequential perceptual prediction induces roughly somatotopically organized representations within the most posterior portion of the frontal lobes, the lateral premotor cortex (PMC) (Schubotz and von Cramon, 2001a). This assumption goes beyond the classical view that the lateral PMC is a crucial component in sensorimotor mapping, i.e., the stepwise transformation of visual target coordinates from a retinocentric reference frame to a body-centered reference frame (Wise et al., 1996). It also goes beyond a more recent model of a premotor "mirror system," ascribing the mapping of observed actions onto action schemes to the ventrolateral PMC (Rizzolatti and Arbib, 1998).

In contrast, it states that any kind of sequential perceptual event, not only observed action, is transformed into a somatotopically organized representation within the lateral PMC whenever its sequential structure is analyzed. Two core hypotheses concerning sequential prediction result from the considered model.

1. Premotor activation increases with the sequential complexity of the attended stimulus sequence.

2. The location of activation within lateral PMC differs according to the motor effector that would be best adapted to be sensory guided by the attended stimulus, even if no movements are performed.

Motivated by these two issues, this study used fMRI to investigate the premotor correlates of predicting visual and auditory sequential events of varying sequential complexity.

Sequences of visual and auditory stimuli were presented in two separate blocks. The complexity of the stimulus sequences was manipulated within both the visual and the auditory block to cause different demands in three experimental conditions ( $L$, low; $M$, middle; $\mathrm{H}$, high; see Methods and Fig. 1). Participants were instructed to analyze the structure of the presented stimulus sequences and to thereby set up perceptual expectations about the ongoing stimulus train. Performance was tested by a forced-choice response 
a Visual Tasks

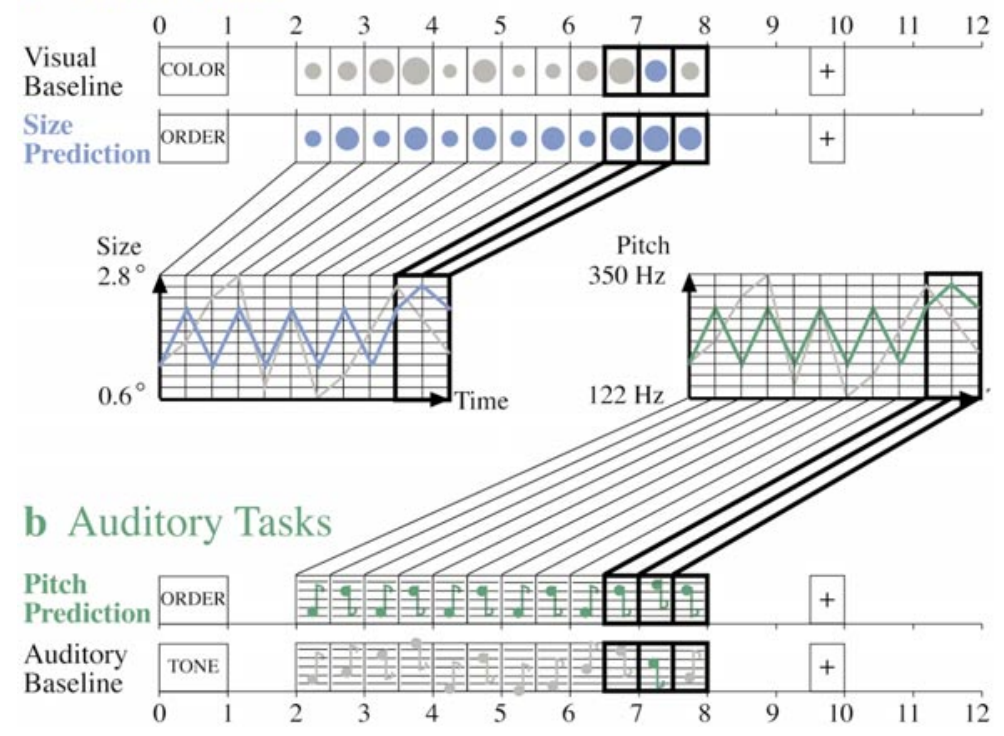

FIG. 1. Experimental tasks. Trial examples are shown for a sequential prediction condition with middle sequential complexity and the baseline condition, each for (a) the visual and (b) the auditory modality. Trials were announced by verbal cues, and after stimulus presentation, a feedback symbol indicated correct ("+") or incorrect ("-") answers. In order to induce prediction in the sequence tasks, participants were asked to build up expectations about the last three stimuli of a trial (as highlighted). In the case of successful prediction, participants were able to indicate whether any stimuli deviated from the sequential pattern introduced by the first nine stimuli within a trial ( $50 \%$ of all presented trials). In contrast, sequential order was irrelevant to indicate deviants in color (visual) or tone quality (auditory), as required in the baseline conditions. All trial examples shown above contain a deviant stimulus on the 11th position.

after the end of the sequence presentation, for which subjects had to decide whether the last three stimuli within the trial matched the expected sequential order. In contrast, sequential structures were irrelevant in a baseline condition, in which subjects were required to indicate predefined targets.

The presented sequences of visual stimuli varied in size, resulting in the perceptual impression of a contracting and expanding motion of a centrally presented disc. According to the assumption of a body-centered representation, predicting systematic changes of stimulus size in a visual stimulus sequence was expected to induce the representation of a sequential hand grip configuration, reflected by activations within premotor areas involved in hand action. The presented sequences of auditory stimuli varied in pitch, resulting in the impression of an isochronously paced tonal pattern. In contrast to the visual condition, predicting systematic changes of tone pitch in an auditory stimulus sequence was expected to induce the representation of a sequential laryngeal configuration, reflected by activations within premotor areas involved in vocal (phonetary) action. In both blocks, stimulation was provided without requiring any concurrent pursuit eye (or other) movements.

We show that analyzed and predicted visual and auditory stimulus sequences are represented within distinct lateral premotor areas and that the pattern of this functional-anatomical dissociation is in line with a rough somatotopical representation. We take these findings to indicate that the prediction of recognized patterns in sequential events involves representations of motor behavior that can be guided by or adapted onto those stimuli. This generalizes the idea of a premotor "mirror system" especially adapted onto the comprehension of observed actions. In contrast to this view, we argue that the lateral PMC role in sequential representation applies on a broader range of perceptions as previously assumed, including both external (observed) actions and any kinds of sequential events.

\section{METHODS \\ Participants}

Fourteen right-handed, healthy volunteers (6 female, mean age 24.8) participated in the study. After being informed about potential risks and screened by a physician of the institution, subjects gave informed consent before participating. The experimental standards were approved by the local ethics committee of the University of Leipzig. Data were handled anonymously.

\section{Procedure}

Participants were instructed and trained in a few days before the MRI experiment. In the MRI session, 


\section{Visual Sequences}
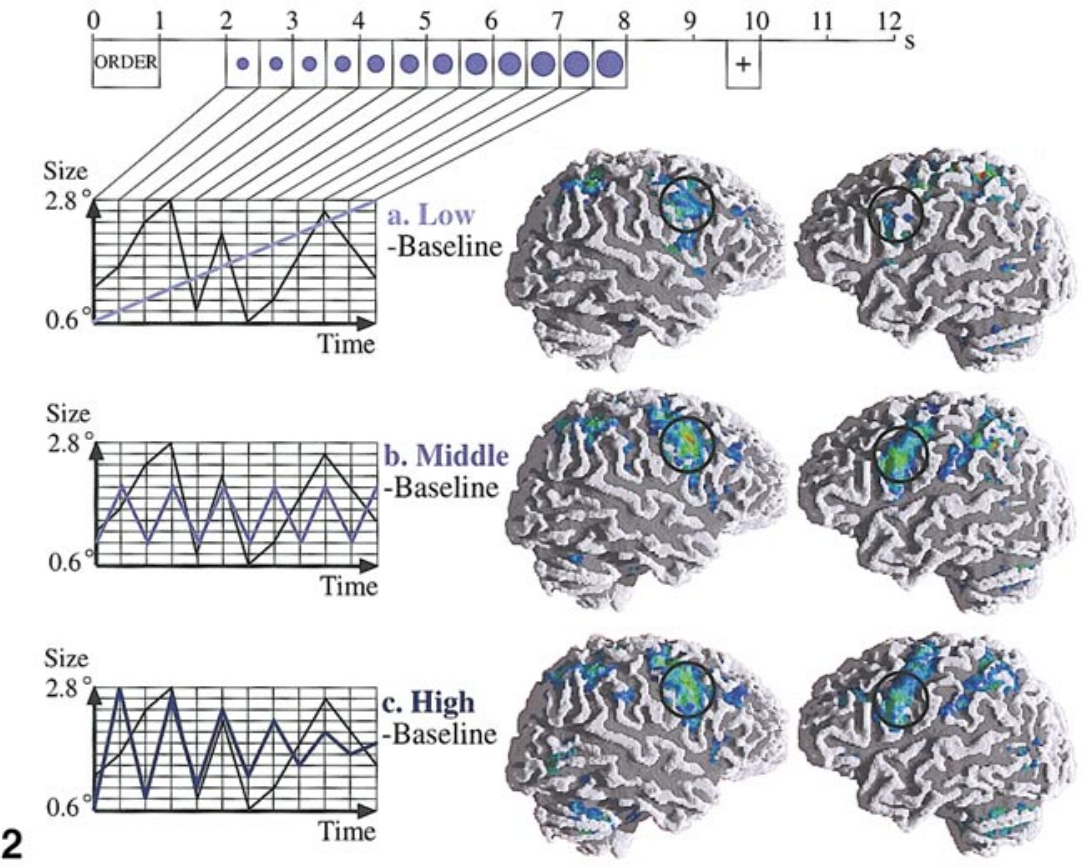

\section{Auditory Sequences}
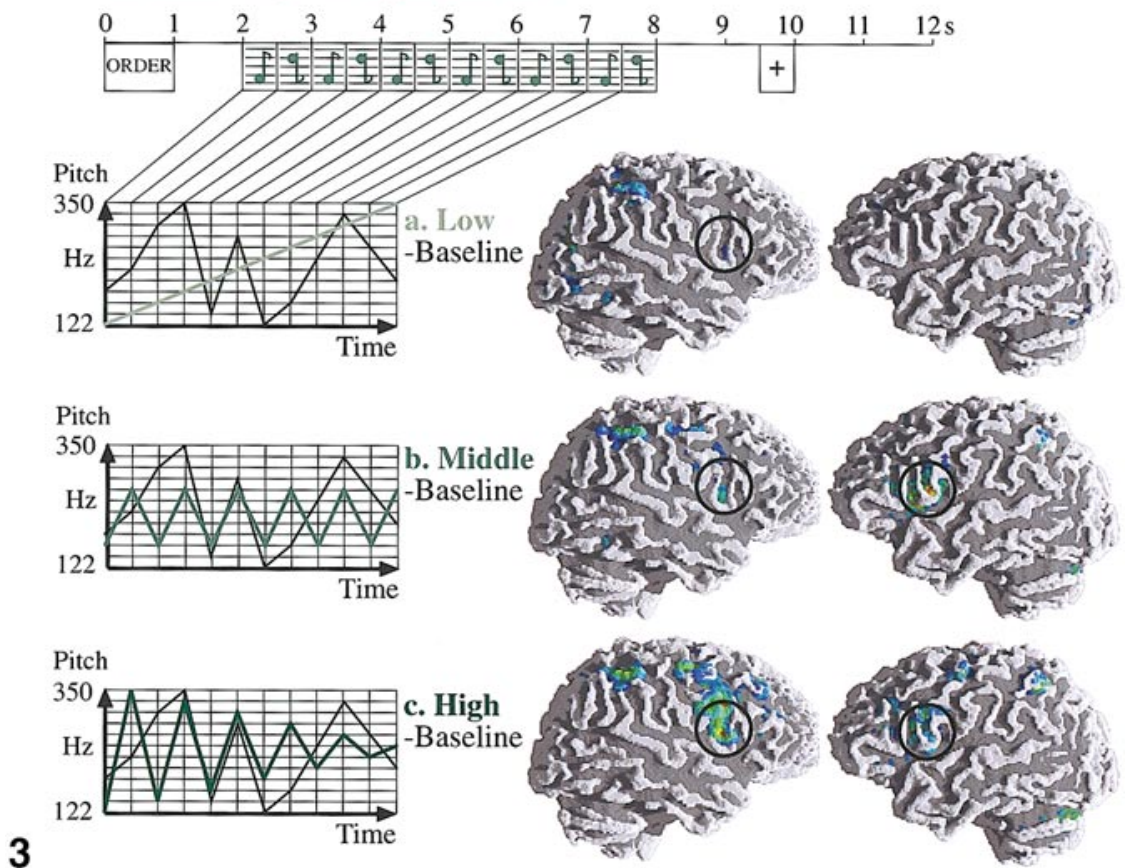

FIG. 2. Effects of sequential complexity in predicting size. Right: Group-averaged brain activations during prediction of visual size sequences relative to the baseline condition are superimposed onto a T1-weighted individual brain. In all images, voxels exceeding a threshold of $Z=3.01$ are shown against a white matter segmentation with partially filled sulci. Circles indicate the superior ventrolateral premotor cortex, the premotor region in which all visual contrasts exposed highest $Z$ scores, increasing from conditions with low (top) to high (bottom) sequential complexity. Top: Trial example of size prediction conditions. Left: Examples of each level of complexity, L, M, and $H$, are displayed as size $\times$ time functions. Each diagram shows the succession of 12 stimuli within one trial. The size of each of the presented circles, ranging from 0.6 to $2.8^{\circ}$ of visual angle, is plotted on the $y$ axis, the time of presentation within the trial is plotted on the $x$ axis (500 ms for each stimulus). The black graphs represent examples of a visual baseline trial, the blue graphs represent examples of each level of complexity of the visual prediction task.

FIG. 3. Effects of sequential complexity in predicting pitch. Right: Group-averaged brain activations ( $Z \geq 3.01)$ during prediction of auditory pitch sequences relative to the baseline condition are superimposed onto a T1-weighted individual brain. Circles indicate the inferiormost ventrolateral premotor cortex, the premotor region in which all auditory contrasts exposed highest $Z$ scores, increasing from conditions with low (top) to high (bottom) sequential complexity. Top: Trial example of pitch prediction conditions. Left: Examples of each level of complexity, L, M, and H, are displayed as pitch $\times$ time functions. The pitch of each of the presented tones, ranging from 122 to 350 $\mathrm{Hz}$, is plotted on the $y$ axis, the time of presentation on the xaxis. The black graphs represent examples of an auditory baselinetrial, the green graphs represent examples of each level of complexity of the auditory prediction task. 
subjects were supine on the scanner bed with their right index and middle fingers positioned on the re sponse buttons. In order to prevent postural adjustments, the subject's arms and hands were carefully stabilized by tape. In addition, form-fitting cushions were used to prevent arm, hand, and head motion. Participants were provided with earplugs to attenuate scanner noise. I mmediately prior to the functional imaging session, subjects spent $20 \mathrm{~min}$ in the scanner, so that they could acclimate to the confinement and sounds of the MR environment.

\section{Stimuli and Tasks (See Fig. 1)}

The stimulus material consisted of 12 circles with diameters ranging from 0.6 to $2.8^{\circ}$ of visual angle (visual conditions) and of 12 tones with a pitch ranging from 122 to $350 \mathrm{~Hz}$ (auditory conditions). In both a visual and an auditory block, three prediction conditions of different sequential complexity (Iow (L), middle $(\mathrm{M})$, and high $(\mathrm{H})$ ) and one baseline condition $(\mathrm{B})$ were presented in a mixed-trial design. Within each trial, 12 stimuli were presented successively at a rate of $500 \mathrm{~ms}$ without temporal gaps, announced by a preceding task cue at the beginning of each trial and followed by a response feedback (see Fig. 1). Stimulus-stimulus transitions differed both within the magnitude of transition in pitch or size and within the direction of transition. By the mean number of different stimulus-stimulus transitions within a trial, we defined the sequential complexity of a condition as low, middle, or high. In the sequential prediction conditions, trials were announced by the cue "order," indicating that participants were required to attend to the sequential order of size (visual) or of pitch (auditory). The participants' task was to judge whether the last three stimuli within a trial matched the stimuli they expected (as indicated in Fig. 1). N ote that the task was perceptual, such that motor requirements were restricted to one single button press after the stimulus sequence in each trial and condition. In contrast, condition B was announced by the cue "color" (visual) or "tone" (auditory), indicating that participants were required to attend to the col or (visual) or the tone quality (auditory). In 50\% of all trials, 1 of the last 3 stimuli was deviant. In the sequential prediction conditions $L, M$, and $H$, deviant stimuli were those which did not match the sequential pattern of the first 9 stimuli, i.e., which were unexpected in size (visual) or pitch (auditory). In condition $B$, deviant stimuli differed in color (visual) or in spectral tone quality (auditory) from all preceding stimuli within the trial. In contrast to the sequential prediction conditions, stimuli were presented in randomized (nonsystematic) order in condition B. Since the sequential order of the first 9 stimuli was irrelevant for identifying deviants in $B$, any sequential patterns could be entirely ignored in this condition. In all conditions, performance was tested by a forced-choice response at the end of each trial (deviant, right index finger; no deviant, right middle finger). There were as many matching as nonmatching targets in each condition, and both occurred with the same frequency in the sequential prediction and in the baseline condition. Accordingly, participants were required to respond for each trial with the same frequency in the prediction and the baseline conditions. Visual and auditory blocks were counterbalanced across subjects.

\section{Imaging}

I maging was performed at $3 \mathrm{~T}$ on a Bruker Medspec $30 / 100$ system equipped with the standard birdcage head coil. Slices were positioned parallel to the bicommissural plane (AC-PC), with 16 slices (thickness 5 $\mathrm{mm}$, spacing $2 \mathrm{~mm}$ ) covering the whole brain. A set of 2D anatomical images was acquired for each subject immediately prior to the functional experiment, using a MDEFT sequence $(256 \times 256$ pixel matrix). Functional images in plane with the anatomical images were acquired using a single-shot gradient EPI sequence $(T E=30 \mathrm{~ms}, 64 \times 64$-pixel matrix, flip angle $90^{\circ}$, field of view $192 \mathrm{~mm}$ ) sensitive to BOLD contrast. During each trial, six images were obtained from 16 axial slices each at the rate of $2 \mathrm{~s} / \mathrm{image}$. In a separate session, high-resolution whole brain images were acquired from each subject to improve the localization of activation foci using a T1-weighted 3D segmented MDEFT sequence covering the whole brain.

\section{Data Analysis}

The MRI data were processed using the software package LI PSI A (Lohmann et al., 2002). In the preprocessing, low-frequency signals (frequencies due to global signal changes like respiration) were suppressed by applying a $1 / 120 \mathrm{~Hz}$ highpass filter. Because low frequencies were removed, temporal filtering also effected a signal control correction. The increased autocorrelation caused by the filtering was taken into account during statistical evaluation by the adjustment of the degrees of freedom. To correct for the temporal offset between the slices acquired in one image, a sincinterpolation algorithm based on the Nyquist Shannon Theorem was employed. To correct for movements, the images of the fMRI time series were geometrically aligned using a matching metric based on linear correlation. The anatomical registration was done in three steps: First, the anatomical slices geometrically aligned with the functional slices were used to compute a transformation matrix, containing rotational and translational parameters, that registered the anatomical slices with the $3 \mathrm{D}$ reference $\mathrm{T} 1$ data set. In a second step, each individual transformation matrix was scaled to the standard Talairach brain size (Talairach and Tournoux, 1988) by applying a linear scal- 
TABLE 1

Talairach Coordinates and Z Scores of Lateral Premotor Activation in Predicting Size and Pitch in Stimulus Sequences of Low, Middle, and High Complexity

\begin{tabular}{|c|c|c|c|c|c|c|c|c|c|c|c|c|}
\hline & \multicolumn{4}{|c|}{ vPMinf (BA6/44) } & \multicolumn{4}{|c|}{ vPMsup (BA6) } & \multicolumn{4}{|c|}{ dPM } \\
\hline & $x$ & $y$ & z & Z score & $x$ & $y$ & z & Z score & $x$ & $y$ & z & Z score \\
\hline \multicolumn{13}{|c|}{ Pitch/auditory } \\
\hline \multirow[t]{2}{*}{ Low } & & & & n.s. & & & & & & & & \\
\hline & 50 & 10 & 3 & 3.1 & & & & & & & & \\
\hline \multirow[t]{2}{*}{ Middle } & -44 & 8 & 9 & 4.6 & & & & & 46 & 3 & 39 & 3.8 \\
\hline & 49 & 4 & 7 & 3.7 & & & & & & & & \\
\hline \multirow[t]{2}{*}{ High } & -47 & 11 & 8 & 4.0 & & & & & 46 & -3 & 40 & 3.4 \\
\hline & 52 & 10 & 5 & 4.5 & & & & & & & & \\
\hline \multirow[t]{2}{*}{ All } & 49 & 7 & 3 & 4.6 & & & & & & & & \\
\hline & -47 & 11 & 7 & 4.4 & & & & & & & & \\
\hline \multicolumn{13}{|l|}{ Size/visual } \\
\hline \multirow[t]{2}{*}{ Low } & & & & & -46 & 3 & 13 & 4.0 & & & & \\
\hline & & & & & 49 & 5 & 24 & 4.5 & & & & \\
\hline \multirow[t]{2}{*}{ Middle } & & & & & -53 & 6 & 15 & 4.9 & & & & \\
\hline & & & & & 46 & 9 & 26 & 5.3 & & & & \\
\hline \multirow[t]{2}{*}{ High } & & & & & -52 & 6 & 15 & 5.7 & & & & \\
\hline & & & & & 47 & 8 & 20 & 5.1 & 31 & 2 & 48 & 5.0 \\
\hline \multirow{2}{*}{ All } & & & & & 43 & 8 & 23 & 5.3 & & & & \\
\hline & & & & & -51 & 3 & 21 & 4.9 & & & & \\
\hline
\end{tabular}

Note PM, premotor cortex; v, ventrolateral; d, dorsolateral; inf, inferior; sup, superior; BA, Brodmann area.

ing. Finally, these normalized transformation matrices were applied to the individual functional raw data. Slice gaps were scaled using a trilinear interpolation, generating output data with a spatial resolution of $3 \mathrm{~mm}^{3}$.

The statistical analysis was based on a least-squares estimation using the general linear model for serially autocorrelated observations (random-effects model) (Friston, 1994; Worsley and Friston, 1995; Zarahn et al., 1997). The design matrix was generated with a boxcar function model and a response delay of $6 \mathrm{~s}$. For each condition, the brain activations during the sequential stimulus presentation were analyzed, except for the first four stimuli (2 s), because this was the minimal number of stimuli required to recognize the sequential pattern and thus to start a sequential prediction. The model equation, including the observation data, the design matrix, and the error term, was convolved with a Gaussian kernel of dispersion of $4 \mathrm{~s}$ FWHM. Within this model, the temporal autocorrelation and the effective degrees of freedom were estimated ( $d f=341$ ). In the following, contrast maps, i.e., estimates of the raw-score differences between specified conditions, were generated for each subject. As the individual functional data sets were all aligned to the same stereotactic reference space, a group analysis was subsequently performed. A one-sample t test of contrast maps across subjects was computed to indicate whether observed differences between conditions were significantly distinct from zero ( $Z \geq 3.09$ ) ( $H$ olmes and Friston, 1998).

\section{RESULTS}

A repeated-measures ANOVA on error rates with the three-level factor Complexity (low, middle, high) yielded a main effect $(F(2,26)=148.3, P<0.0001)$ indicating significantly different error rates in the conditions L (2.9\%), M (13.1\%), and H (27.5\%), across both dimensions. These behavioral effects were reflected by the anatomical results. Relative to $B$, all sequential prediction types elicited significant activations within the lateral PMC (see Table 1) and further cortical areas (Table 2). As shown in Figs. 2 and 3, the maximal $Z$ scores of premotor activation foci covaried positively with the sequential complexity of stimulus trains in both the visual and the auditory conditions. As shown in Fig. 4, the perceptual dimension of the stimulus sequences had a clear effect on the core center of premotor activation. Thus, the visually induced activations showed highest $Z$ scores within the superior ventrolateral PMC (vPMsup), whereas auditorily induced activations yielded highest $Z$ scores within the transition from BA 6 to BA 44, in the inferior ventrolateral PMC (vPMinf). These two areas exposed distinct clusters of activations with maximal $Z$ scores for the visual and the auditory conditions.

In order to confirm both the effect of sequential complexity and the effect of stimulus dimension, a statistical analysis of anatomical regions of interest (ROIs) was computed (Bosch, 2000). To this end, four spherical ROIs with a radius of $5 \mathrm{~mm}$ each were defined for right and left vPMsup and for right and left vPMinf. 
TABLE 2

Talairach Coordinates and Z Scores of Further Activation in Predicting Size and Pitch

\begin{tabular}{|c|c|c|c|c|c|c|c|c|c|c|c|c|}
\hline & \multicolumn{4}{|c|}{ Low } & \multicolumn{4}{|c|}{ Middle } & \multicolumn{4}{|c|}{ High } \\
\hline & $x$ & $y$ & $z$ & Z score & $x$ & $y$ & z & Z score & $x$ & $y$ & z & Z score \\
\hline \multicolumn{13}{|c|}{ Pitch/auditory } \\
\hline SMA & & & & & -2 & -1 & 51 & 3.6 & -5 & 1 & 53 & 3.9 \\
\hline IPS & & & & & -47 & -43 & 45 & 4.0 & -47 & -40 & 42 & 4.4 \\
\hline & 46 & -36 & 46 & 3.7 & 46 & -30 & 45 & 4.6 & 46 & -32 & 49 & 4.2 \\
\hline FG/CE & & & & & -27 & -67 & -14 & 3.6 & -23 & -67 & -14 & 4.1 \\
\hline \multicolumn{13}{|l|}{ Size/visual } \\
\hline SMA & & & & & 4 & 15 & 44 & 4.0 & -11 & 18 & 43 & 4.3 \\
\hline \multirow[t]{4}{*}{ IPS } & -44 & -36 & 35 & 4.0 & -56 & -29 & 36 & 4.6 & -50 & -27 & 33 & 4.7 \\
\hline & 46 & -36 & 46 & 4.4 & 46 & -36 & 46 & 4.3 & 46 & -35 & 38 & 4.3 \\
\hline & 31 & -45 & 40 & 4.8 & 31 & -45 & 40 & 4.8 & 37 & -44 & 43 & 4.3 \\
\hline & & & & & -44 & -40 & 36 & 5.0 & -41 & -43 & 40 & 4.2 \\
\hline \multirow[t]{2}{*}{$\mathrm{FG} / \mathrm{CE}$} & & & & & -29 & -65 & -18 & 4.1 & -29 & -62 & -18 & 4.7 \\
\hline & & & & & & & & & 40 & -62 & -18 & 4.2 \\
\hline
\end{tabular}

Note SMA, supplementary motor area; IPS, intraparietal sulcus; FG, fusiform gyrus; CE, cerebellar cortex.

The exact anatomical locations of the ROIs were established as follows. Two new group $z$ maps were generated which resulted from contrasting all levels of sequential complexity $(L, M$, and $H)$ against the baseline $B$ for the visual conditions (Vis-B) and for the auditory conditions (Aud-B), respectively. In each contrast, Vis-B and Aud-B, the coordinates of maximal activation within the lateral PMC were then extracted (see Table 1). As expected, local maxima were located within the VPMsup for the contrast Vis-B and within the vPMinf for the contrast Aud-B. The ROIs for the vPMsup were then centered on the local maxima in Vis-B and the ROIs for the vPMinf on those in Aud-B. As apparently both areas were activated bilaterally in nearly all conditions, activations were subsequently collapsed over hemispheres. For all voxels within a ROI, a mean contrast value was then calculated for each dimension (visual, auditory), each complexity

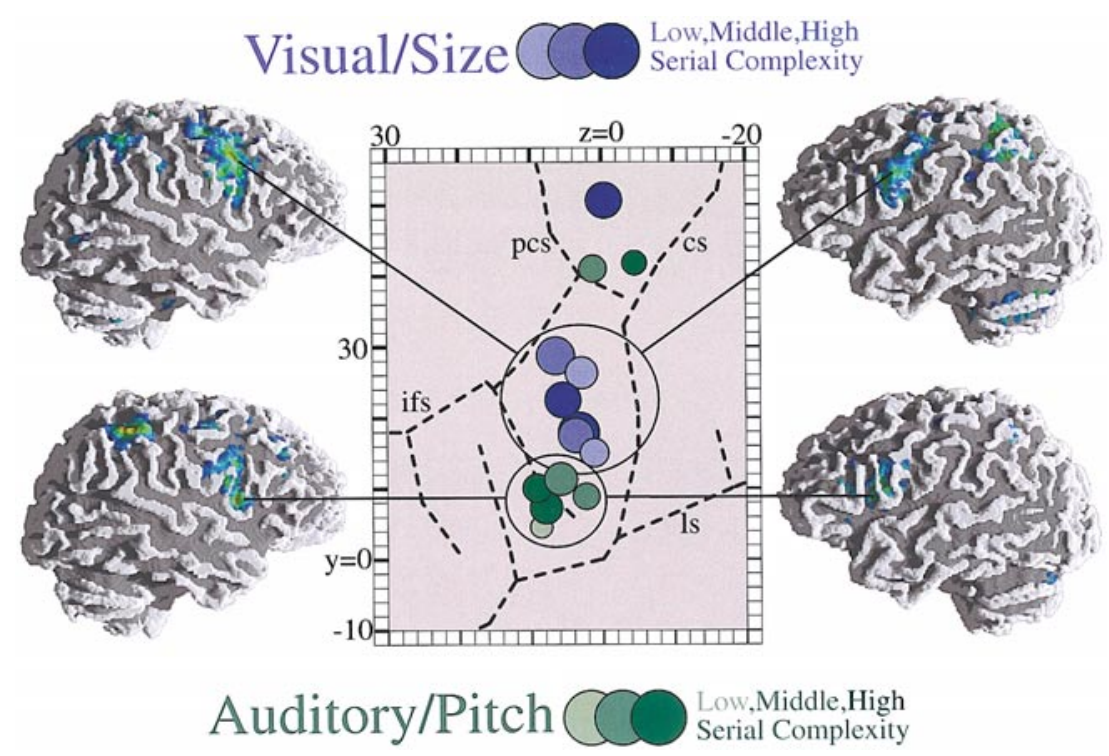

FIG. 4. Somatotopical activation pattern for predicting size and pitch. The white matter segmentations show the group-averaged brain activations for all visual conditions versus basel ine (on the top) and for all auditory conditions versus baseline (on the bottom). Central graph: Premotor activation foci with highest $Z$ scores from group-averaged data in the visual (blue) and the auditory (green) conditions are collapsed from the left and the right hemispheres. The color intensities of the foci indicate the sequential complexities of the conditions (low, middle, high). The relative diameter of each focus indicates the corresponding Z score of the activation, as listed in Table 1. Black dotted lines indicate the locations of the inferior frontal sulcus (ifs), the precentral sulcus (pcs), the lateral sulcus (Is), and the central sulcus (cs) within an individual brain. Talairach coordinates of the $y$ plane (anterior-posterior) and the $z$ plane (superior-inferior) are indicated, the $x$ plane (right-left) is flattened. 
(low, middle, high), and each subject. These mean values subsequently entered a repeated-measures ANOVA with the two-level factor ROI (vPMsup, vPMinf), the two-level factor Dimension (visual, auditory), and the three-level factor Complexity (low, middle, high).

This ANOVA yielded, first, a main effect of Complexity $(F(2,26)=29.4, P<0.0001)$ showing that activations in the lateral PMC differed significantly between levels of complexity $L, M$, and $H$. This statistically confirmed that premotor activation increased with sequential complexity of the attended stimulus train both within the visual conditions (Fig. 2) and within the auditory conditions (Fig. 3). Second, there was a significant interaction of ROI $\times$ Dimension $(F(1,13)=$ 10.8, $\mathrm{P}<0.006)$, showing that activation differences between vPMsup and vPMinf were significantly different within the visual and within the auditory dimension, respectively. This statistically confirmed that the location of activation within the lateral PMC differed significantly between the visual and the auditory conditions (Fig. 4).

\section{DISCUSSION}

The present findings indicate that premotor activation increases with the sequential complexity of a predicted stimulus sequence and that the focus of that premotor activation differs according to the perceptual information to be predicted. The functional-anatomical dissociation implicates a correspondence between target size prediction and the vPMsup and between target pitch prediction and the vPMinf.

As will be discussed in the following, these premotor areas have also been found to be crucial in the movements of different motor effectors, with the vPMsup to be more prominent in hand movements and the VPM inf to be more prominent in laryngeal movements. There fore, we take this outcome as evidence of our hypothesis that an arbitrary stimulus sequence induces a somatotopical representation within the lateral PMC whenever its sequential structure is analyzed. This suggests that there might be an immediate link between the prediction of upcoming events and a representation of corresponding motor schemas or motor imageries.

\section{Predicting Size Modulates Premotor Areas Involved in $\mathrm{H}$ and Movement}

Predicting visual size in sequential events elicited most significant activations within the vPMsup, the portion of BA 6 that is posteriorly adjacent to the junction of the inferior precentral sulcus and the inferior frontal sulcus (Fig. 2). The functional importance of this area in object-hand action like object grasping or object manipulation has been repeatedly confirmed in humans by PET and FMRI (Decety et al., 1994; Martin et al., 1995; Matsumura et al., 1996; Grafton et al., 1996, 1997; Gruenewald et al., 2000). These imaging data parallel findings in the macaque that indicate a ventral premotor area to be especially adapted to the hand- object mapping (area F5). Thus, area F 5 exposes a selectivity for manual grip types (Gentilucci et al., 1988; J eannerod et al., 1995) and responds to both observed objects (Murata et al., 1997; Rizzolatti et al., 1988) and observed hand-object action (Rizzolatti et al., 1996; Rizzolatti and Fadiga, 1998). To account for the latter finding, the notion of an "action observation/ execution matching system" or mirror system has recently emerged (Rizzolatti and Arbib, 1998). It states that area F5 mediates a matching of observed actions onto concepts of action, a process which is suggested to underlie the understanding of observed actions. Similar functions are now suggested for the human ventrolateral PMC, as well, extending its role in hand- object action from self-performed execution to perceptual analysis (observation) of manual action (I acoboni et al., 1999; Buccino et al., 2001).

Although the present study neither employed real objects nor required the execution or the observation of object-related actions, it yielded findings which we believe to be particularly significant for a broader understanding of premotor functions in humans. Thus, strikingly similar ventrolateral premotor activations as found during the observation and execution of handobject actions were also caused by the extraction of predictable size patterns in sequences of abstract visual stimuli. Likewise, the sequential prediction of form properties in the same type of stimuli has shown to yield vast activations within the MPM (Schubotz and von Cramon, 2001a).

From that we may ask what might be functionally common to the prediction of sequential events and the observation (and thereby understanding) of action. A straightforward explanation might be that attending to sequential events and attending to external actions both induce some fundamentally equivalent processes. Although most goal-directed actions allow for certain variations, their basic sequential schedule, their "syntax," is fixed and mandatory, as it is in predefined stimulus sequences. Thus, both abstract stimulus sequences that follow a predefined pattern (as employed in the present study) as well as standard goal-directed actions are sequential events that are relatively well predictable. Valid rules, based on which the course of sequential events is classified as being expected or unexpected, can obviously be acquired and applied in observed actions as well as in any abstract stimulus sequences. The notion of understanding refers to the same behavioral effect in the context of action observation and event observation: the observer who has found out sequential regularities is able to respond appropriately to ongoing events or observed actions. 
According to these suggestions and the present imaging data, it appears that the lateral PMC is adapted to the analysis of meaningful patterns in any kind of sequential events, including the special case of observed actions. Certainly, the present study cannot settle what kind of representation is reflected by premotor activations in perceptual prediction. This might be a motor scheme (motor plan, motor imagery), a sensory representation of an observed target, or, finally, a sensorimotor representation that is neither purely sensory nor purely visual, as suggested for some neurons in the monkey PMv (Fadiga et al., 2000). Likewise, our findings cannot settle whether we predict events by motor planning or imagery because we imagine motor acts by predicting the sensory feedback they effect, i.e., by sensory events. These issues remain to be investigated in future studies.

\section{Predicting Pitch Modulates Premotor Areas Involved in Articulation}

Predicting auditory pitch in sequential events elicited most significant activations in the inferiormost ventrolateral part of PMC directly adjacent to and extending into the more anterior region of BA 44 (Broca's area) (Fig. 3). This cortex is suggested in the processing of sequential sounds (Platel et al., 1997) and chords (Maess et al., 2001) and of visually as well as auditorily mediated rhythms (Schubotz \&t al., 2000; Schubotz and von Cramon, 2001a,b) and in the discrimination of variations in pitch patterns (Gandour et al., 2000). As proposed for all lateral premotor areas, it has been emphasized that Broca's area recognizes functional (meaningful) properties, rather than simple acoustic properties, of complex auditory cues (Gandour et al., 1998). For the present findings, it is particularly interesting that BA 44 mediates behaviors that require the mapping of an auditory signal onto an articulatory motor plan. Thus, Broca's area is suggested to map sound onto pronunciation in vocal imitation (Skoyles, 1998; Kuhl and Meltzoff, 1992). These findings are in line with the present results, which indicate that a pitch prediction is reflected by a mapping from sound to vocal plans or the imagination of vocal production.

\section{H igher Complexity Additionally Modulates Premotor Regions of Far Space Coding}

Increasing sequential complexity also caused some weaker, though significant activation foci within the dorsolateral premotor regions for both perceptual dimensions (Table 1 and Fig. 4). This area is known to mediate far-space coding (Anderson et al., 1993; Rizzolatti et al., 1994; Petit et al., 1996; Boussaoud, 1995) and has recently been implicated in merging both near and far space (I acoboni et al., 1997). In fact, both visual and auditory stimuli presented in our study could be conceived of as inducing the perceptual impression of an approaching and departing motion. For the visual dimension, a change of relative disparity is a sufficient binocular stimulus for the perception of motion in depth (Regan et al., 1995). For the auditory dimension, a popular illusion associated with the Doppler effect is that the sound source approaches as the pitch rises (Neuhoff and McBeath, 1996). Accordingly, the impression of stimuli that move out of reach into far space and return into near space might have been induced in conditions with higher sequential complexity (conditions $\mathrm{M}$ and $\mathrm{H}$ ). Therefore, premotor areas that code far space and/or mediate between near and far space could be expected to be more involved in more complex conditions.

\section{CONCLUSION}

The present findings show that, in the absence of any motor requirements, prediction of perceived sequential events induces representations within the lateral PMC that differ according to the stimulus dimension. Moreover, the anatomical distribution of activations induced by different stimulus types suggests that their representations are organized al ong the rough somatotopical organization that has been attributed to the monkey and the human PMC. Extracting and predicting the sequential structure of a stimulus train yielded core activations within the premotor areas involved in hand movement for systematic size modulations in a visual stimulus sequence and within the premotor areas involved in vocal movement for systematic pitch modulations in an auditory stimulus sequence. This somatotopical pattern points to a functional architecture that serves the immediate mapping of perception onto action and vice versa. While this proposal is in line with the concept of a premotor mirror function, our findings indicate that also abstract sequences of perceptual events, not only observed actions, are transformed into a motor representation within the lateral PMC whenever its sequential course has to be analyzed and predicted. We believe that our findings, together with other considered data, contribute to a more comprehensive model of perception-action mapping in the human neocortex.

\section{ACKNOWLEDGMENTS}

We thank Marcel Brass, Evelyn Ferstl, Sonja Kotz, Burkhard Maess, Sophie Manthey, Markus Ullsperger, and Thomas J acobsen for hel pful comments; Stefan Zysset, Gaby Lohmann, Volker Bosch, and Karsten Müller for support in MRI statistics; and Katrin Sakreida for experimental assistance.

\section{REFERENCES}

Anderson, R. A., Snyder, L. H., Li, C. S., and Stricanne, B. 1993. Coordinate transformations in the representation of spatial information. Curr. Opin. Neurobiol. 3: 171-176. 
Bosch, V. 2000. Statistical analysis of multi-subject fMRI data: The assessment of focal activations. J . Magn. Reson. Imaging 11: 6164.

Boussaoud, D. 1995. Primate premotor cortex: Modulation of preparatory neuronal activity by gaze angle. J . Neurophysiol. 73: 886890.

Buccino, G., Binkofski, F., Fink, G. R., Fadiga, L., Fogassi, V., Gallese, R., Seitz, R. J ., Zilles, K., Rizzolatti, G., and Freund, H.-J . 2001. Action observation activates premotor and parietal areas in a somatotopic manner: An fMRI study. Eur. J . Neurosci. 13: 400404.

Decety, J ., Perani, D., J eannerod, M., Bettinardi, V., Tadary, B., Woods, R., Mazziotta, J . C., and Fazio, F. 1994. Mapping motor representations with positron emission tomography. Nature 371: 600-602.

Fadiga, L., Fogassi, L., Gallese, V., and Rizzolatti, G. 2000. Visuomotor neurons: Ambiguity of the discharge or 'motor' perception? Int. J . Psychophysiol. 35: 156-177.

Friston, K. 1994. Statistical parametric maps in functional imaging: A general linear approach. Hum. Brain Mapping 2: 189-210.

Gandour, J ., Wong, D., Hsieh, L., Weinzapfel, B., Van Lancker, D., and Hutchins, G. D. 2000. A crosslinguistic PET study of tone perception. J . Cognit. Neurosci. 12: 207-222.

Gandour, J ., Wong, D., and Hutchins, G. 1998. Pitch processing in the human brain is influenced by language experience. NeuroReport 9: 2115-2219.

Gentilucci, M., Fogassi, L., Luppino, G., Matelli, M., Camarda, R. M., and Rizzolatti, G. 1988. Functional organization of inferior area 6 in the macaque monkey. I. Somatotopy and the control of proximal movements. Exp. Brain Res. 71: 475- 490.

Grafton, S. T., Arbib, M. A., Fadiga, L., and Rizzolatti, G. 1996. Localization of grasp representation in humans by PET. Exp. Brain Res. 112: 103-111.

Grafton, S. T., Fadiga, L., Arbib, M. A., and Rizzolatti, G. 1997. Premotor cortex activation during observation and naming of familiar tools. Neurol mage 6: 231-236.

Gruenewald, C., Mecklinger, A., Besson, M., and von Cramon, D. Y. 2000. Motor representation and their role in visual working memory. J . Cognit. Neurosci. Suppl: 143.

Holmes, A. P., and Friston, K. J . 1998. Generalisability, random effects and population inference. Neurol mage 7: 754.

Hommel, B., Müsseler, J ., Aschersleben, G., and Prinz, W. 2001. The theory of event coding (TEC): A framework for perception and action. Behav. Brain Sci., in press.

Iacoboni, M., Woods, R. P., Lenzi, G. L., and Mazziotta, J . C. 1997. Merging of oculomotor and somatomotor space coding in the human right precentral gyrus. Brain 120: 1635-1645.

Iacoboni, M., Woods, R. P., Brass, M., Bekkering, H., Mazziotta, J. C., and Rizzolatti, G. 1999. Cortical mechanisms of human imitation. Science 286: 2526-2528.

Ingvar, D. H. 1985. "Memory of the future": An essay on the temporal organization of conscious awareness. Hum. Neurobiol. 4: 127-136.

J eannerod, M., Arbib, M. A., Rizzolatti, G., and Sakata, H. 1995. Grasping objects: The cortical mechanisms of visuomotor transformation. Trends Neurosci. 18: 314-320.

Kawashima, R., Tanji, J ., Okada, K., Sugiura, M., Sato, K., Kinomura, S., I noue, K., Ogawa, A., and Fukuda, H. 1998. Oculomotor sequence learning: A positron emission tomography study. Exp. Brain Res. 122: 1-8.

Kuhl, P. K., and Meltzoff, A. N. 1982. The bimodal perception of speech in infancy. Science 218: 1138-1141.

Lekwuwa, G. U., and Barnes, G. R. 1996. Cerebral control of eye movements. II. Timing of anticipatory eye movements, predictive pursuit and phase errors in focal cerebral lesions. Brain 119: 491-505.

Lohmann, G., Mueller, K., Bosch, V., Mentzel, H., Hessler, S., Chen, L., and von Cramon, D. Y. 2001. Lipsia-A new software system for the evaluation of functional magnetic resonance images of the human brain. Comput. Med. Imaging Graph. 25: 449- 457.

Maess, B., Koelsch, S., Gunter, T., and Friederici, A. D. 2001. Musical syntax is processed in Broca's area: An MEG study. Nat. Neurosci. 4: 450- 454.

Martin, A., Haxby, J . V., Lalonde, F. M., Wiggs, C. L., and Ungerleider, L. G. 1995. Discrete cortical regions associated with knowledge of color and knowledge of action. Science 270: 102-105.

Matsumura, M., Kawashima, R., Naito, E., Satoh, K., Takahashi, T., Yanagisawa, T., and Fukuda, H. 1996. Changes in rCBF during grasping in humans examined by PET. NeuroReport 7: 749-752.

Murata, A., Fadiga, L., Fogassi, L., Gallese, V., Raos, V., and Rizzolatti, G. 1997. Object representation in the ventral premotor cortex. J . Neurophysiol. 78: 2226-2230.

Neuhoff, J . G., and McBeath, M. K. 1996. The Doppler illusion: The influence of dynamic intensity change on perceived pitch. J . Exp. Psychol. 22: 970-985.

Patel, A. D., and Balaban, E. 2000. Temporal patterns of human cortical activity reflect tone sequence structure. Nature 404: 8084.

Petit, L., Orssaud, C., Tzourio, N., Crivello, F., Berthoz, A., and Mazoyer, B. 1996. Functional anatomy of pre-learned sequence of horizontal saccades in humans. J . Neurosci. 16: 3714-3726.

Platel. H., Price, C., Baron, J . C., Wise, R., Lambert, J ., Frackowiak, R. S., Lechevalier, B., and Eustache, F. 1997. The structural components of music perception. A functional anatomical study. Brain 120: 229-243.

Regan, D., Hamstra, S. J ., Kaushal, S., Vincent A., Gray, R., and Beverly, K. I. 1995. Visual processing of the motion of an object in three dimensions for a stationary or a moving observer. Perception 24: 87-103.

Rizzolatti, G., and Arbib, M. A. 1998. Language within our grasp. Trends Neurosci. 21: 188-194.

Rizzolatti, G., and Fadiga, L. 1998. Grasping objects and grasping action meanings: The dual role of monkey rostroventral premotor cortex (area F5). Novartis Found. Symp. 218: 81-95.

Rizzolatti, G., Camarda, R., Fogassi, L., Gentilucci, M., Luppino, G., and Matelli, M. 1988. Functional organization of inferior area 6 in the macaque monkey. II. Area F5 and the control of distal movements. Exp. Brain Res. 71: 847- 856.

Rizzolatti, G., Fadiga, L., Gallese, V., and Fogassi, L. 1996. Premotor cortex and the recognition of motor actions. Cognit. Brain Res. 3: 131-141.

Rizzolatti, G., Riggio, L., and Sheliga, B. M. 1994. Space and selective attention. In Attention and Performance XV. Conscious and Nonconscious Information Processing (C. Umilta and M. Moskovitch, Eds.), pp. 231-265. MIT Press, Cambridge, MA.

Schubotz, R. I., and von Cramon, D. Y. 2001a. Functional organization of the lateral premotor cortex: fMRI reveals different regions activated by anticipation of object properties, location and speed. Cognit. Brain Res. 11: 97-112.

Schubotz, R. I., and von Cramon, D. Y. 2001b. Interval and ordinal properties of sequences are associated with distinct premotor areas. Cereb. Cortex 11: 210-222.

Schubotz, R. I., Friederici, D. A., and von Cramon, D. Y. 2000. Time perception and motor timing: A common cortical and subcortical basis revealed by fMRI. Neurol mage 11: 1-12.

Skoyles, J. R. 1998. Speech phones are a replication code. Med. Hypotheses 50: 167-173. 
Talairach, J ., and Tournoux, P. 1988. Co-planar Stereotaxis Atlas of the Human Brain. Thieme, New York.

Wise, S. P., di Pellegrino, G., and Boussaoud D. 1996. The premotor cortex and nonstandard sensorimotor mapping. Can. J . Physiol. Pharmacol. 74: 469-482.

Worsley, K., and Friston, K. 1995. Analysis of fMRI time-series revised-Again. Neurol mage 2: 359-365.
Zarahn, E., Aguirre, G. K., and D'Esposito, M. 1997. Empirical analysis of BOLD fMRI statistics. I. Spatially smoothed data collected under null-hypothesis and experimental conditions. Neurol mage 5: 179-197.

Zhuang, P., Dang, N., Waziri, A., Gerloff, C., Cohen, L. G., Hallett, M., and Waziri, A. 1998. Implicit and explicit learning in an auditory serial reaction time task. Acta Neurol. Scand. 97: 131-137. 